\title{
Swiss Medical Board: cui bono?
}

\author{
Thomas Cerny ${ }^{a}$, \\ Jakob Passweg , \\ Giorgio Nosedac \\ a président d'Oncosuisse \\ b président de la Ligue suisse \\ contre le cancer \\ c ancien président d'Onco- \\ suisse
}

Liens d'intérêts: aucun

Un commentaire du Swiss Medical Board se trouve à la page 1729.

Correspondance:

Prof. Dr Thomas Cerny

Oncosuisse

Effingerstrasse 40

CH-3008 Berne

Tél. 0313899100

Fax 0313899160

thomas.cerny[at]kssg.ch
Les rapports du Swiss Medical Board (SMB) suscitent régulièrement de vives réactions, ce qui n'a rien d'étonnant en soi, car ils abordent des thèmes conflictuels. On a pu le voir avec le rapport sur le dépistage systématique par mammographie publié en février dernier [1] ou avec celui sur l'emploi de statines en prévention primaire des maladies cardiovasculaires, paru en avril [2]. On ne saurait toutefois ramener ces réactions parfois virulentes à une simple défense d'intérêts. La composition du SMB, sa méthode de travail et la démarche adoptée - en d'autres termes, le professionnalisme de l'organisation - sont également sous les feux de la critique, à l'instar du choix des thèmes, respectivement de la façon de les délimiter.

\section{Mêmes données, autres conclusions}

Dans le cadre de son rapport sur le dépistage systématique par mammographie, le SMB recommande de ne pas introduire de nouveaux programmes et de limiter les programmes existants dans le temps. Il conseille par ailleurs d'évaluer toutes les formes de dépistage par mammographie et préconise une consultation médicale préalable approfondie avec des explications claires pour les femmes.

Ce rapport a donné lieu à de violentes critiques. Il n'apporte pas de nouveaux éléments; les études sur lesquelles il se fonde et leurs résultats sont connus depuis de nombreuses années. L'analyse du rapport coût-efficacité effectuée par le SMB ne répond en aucune manière aux exigences auxquelles doivent satisfaire des évaluations d'interventions médicales en général. Il est intéressant de constater que les résultats de ce calcul coût-efficacité n'ont d'ailleurs pas été avancés comme argument par le SMB.

En résumé, nous sommes d'avis que le SMB sous-estime les effets positifs du dépistage systématique par mammographie, alors qu'il en surestime les effets indésirables (voir aussi en encadré l'analyse de $\mathrm{H}$. de Koning). Le groupe d'experts anglais qui a publié en 2012 une revue largement reconnue [3] mettant en lumière de façon très différenciée les avantages et les inconvénients du dépistage systématique par mammographie en place depuis 1988 est arrivé à la conclusion que les programmes apportent un bénéfice important et qu'il convient de les maintenir. Il est intéressant de relever que le SMB cite cette publication comme une base importante pour son propre rapport alors qu'il arrive à des conclusions diamétralement opposées.

En janvier 2014, une semaine avant la parution du rapport du SMB, le Conseil néerlandais de la

\section{Résumé}

Dans un rapport sur le dépistage systématique par mammographie publié en février 2014, le Swiss Medical Board (SMB) arrive à la conclusion que les inconvénients du dépistage organisé l'emportent sur les bénéfices.

Ce rapport a donné lieu à de vives critiques. L'analyse présente en effet de graves lacunes. Dans l'ensemble, les effets positifs du dépistage organisé sont sous-estimés, alors que les conséquences négatives sont surestimées. Le fait de compter ensemble les faux positifs et les surdiagnostics - deux éléments foncièrement différents, on le sait - en les mettant dans la seule et même catégorie des «résultats faussement positifs» illustre bien ces insuffisances. En associant les experts consultés à l'élaboration du rapport, le SMB aurait évité ce type d'erreurs.

Le SMB s'est exprimé sur les critiques formulées dans le cadre d'une Post-Publication Review. La situation demeurant selon lui «inchangée», il maintient ses recommandations.

Force est de constater que le SMB a analysé le dépistage systématique par mammographie de manière partiale. II n'apporte pas de nouvelles données dans son rapport et ne tient pas compte du dépistage opportuniste ni de l'avis des experts consultés. Au lieu de cela, il effectue une analyse coût-efficacité difficilement compréhensible et formule des recommandations contradictoires.

Nous estimons que le dépistage systématique par mammographie doit continuer à être développé à la lumière des avancées scientifiques. Pour ce faire, il est nécessaire de pouvoir s'appuyer sur des bases solides et nuancées - une exigence que le rapport du SMB sur le dépistage systématique par mammographie ne remplit pas.

santé a publié un rapport volumineux sur le dépistage systématique par mammographie aux Pays-Bas [4]. Ce groupe d'experts s'est lui aussi prononcé en faveur du maintien des programmes. 
Les recommandations du SMB sont contradictoires. Si, après une consultation médicale approfondie et des explications claires sur les bénéfices et les risques du dépistage, une femme décide de se faire dépister, la mammographie devrait, si on suit la logique du SMB - c'est-à-dire une fois que l'on aura mis fin aux programmes - être réalisée dans le cadre d'un dépistage opportuniste. Or, on sait parfaitement qu'il est plus difficile de contrôler la qualité dans ce cas. Et pourquoi évaluer toutes les formes du dépistage par mammographie en termes de qualité si les inconvénients l'emportent clairement?

\section{Où sont passés tous les experts?}

La parution d'un rapport du SMB entraîne souvent un chapelet de récriminations, ce qui est surprenant dans la mesure où le règlement relatif à l'organisation et aux processus de cet organe [5] prévoit le recours à des experts externes pour bénéficier de leur savoir spécifique. Les experts ont bien été consultés et ils sont mentionnés dans le rapport. Mais étonnamment, quelques-uns de ces spécialistes ne trouvent leurs commentaires et arguments nulle part dans le rapport [6]. Le SMB n'a en particulier pas repris la suggestion des experts de tenir compte des évaluations réalisées par les programmes. Pour des interventions de santé publique qui s'inscrivent dans une perspective à long terme - et le dépistage

\section{Une Post-Publication Review inutile?}

Depuis peu, le SMB invite organisations et experts à soumettre après coup leur position sur le rapport sous la forme de Post-Publication Reviews.

Les experts consultés (dont l'avis n'apparaît pas dans le rapport, nous l'avons mentionné plus haut) sont donc invités à exprimer encore une fois leur opinion par le biais de ce canal. Cela en vaut-il la peine? Apparemment pas.

La Post-Publication Review parue le 12 août 2014 [7] confirme nos doutes: elle s'inscrit dans le droit fil du rapport initial. A la lecture du condensé, on pourrait presque avoir l'impression que le rapport du SMB a davantage suscité l'adhésion que la critique. Les reproches formulés dans le cadre du débat se résument en effet à cette seule phrase: «L'interprétation des données et les conséquences tirées de cette interprétation donnent notamment lieu à des divergences d'opinion.» Réduire les critiques exprimées quant à la méthode utilisée à une «interprétation des données» nous semble un peu court. L'emploi de l'indice de Karnofsky pour calculer les QALY, par exemple, n'est pas usuel et n'est pas approprié dans ce contexte; que le SMB persiste et signe n'y change rien.

Au vu de ce qui précède, il n'est pas étonnant que l'organe scientifique du SMB maintienne ses recommandations en alléguant une «situation inchangée».

\section{«Les effets positifs du dépistage organisé sont sous-estimés, alors que les conséquences négatives sont surestimées.»}

systématique par mammographie entre dans cette catégorie -, cela constituerait certainement un complément judicieux aux études randomisées (qui datent, dans ce domaine).

Il est également surprenant de voir que la Fédération des programmes de dépistage du cancer, swiss cancer screening, qui a demandé à être biffée de la liste des experts associés à l'élaboration du rapport après avoir constaté que ses avis n'étaient pas pris en compte, est désormais mentionnée dans une note de bas de page en tant qu'«organisation contactée». On attendrait d'un rapport rédigé de façon professionnelle que les avis des experts apparaissent de manière transparente et non qu'ils soient mentionnés ou non selon le bon vouloir des auteurs.

En associant réellement les experts à l'élaboration du rapport, on aurait également pu éviter des erreurs grossières comme le fait de compter ensemble les faux positifs et les surdiagnostics en les mettant dans la seule et même catégorie des «résultats faussement positifs». Le doute profitant à l'accusé, nous partirons du principe que cet amalgame n'est pas intentionnel.
La révision du rapport que certains représentants du SMB avaient laissé entrevoir ne devrait donc pas être pour demain.

\section{Une réforme du SMB s'impose}

Le SMB a fait preuve d'un manque d'objectivité dans l'analyse du dépistage systématique par mammographie. Il n'a pas pris en compte de nouvelles données scientifiques, n'a pas indiqué l'avis des experts consultés et n'a pas tenu compte de la réalité suisse en n'abordant pas la question du dépistage opportuniste largement répandu dans notre pays. Au lieu de cela, il a procédé à une analyse coût-efficacité difficilement compréhensible et formulé des recommandations contradictoires. Ces insuffisances seraient-elles dues à la composition du SMB, qui ne semble pas disposer des connaissances méthodologiques et cliniques nécessaires?

Nous estimons que le dépistage par mammographie doit continuer à être développé et que les actes médicaux doivent, dans toute la mesure du possible, s'appuyer sur des preuves scientifiques. Pour cela, il est toutefois indispensable de disposer de bases de 


\section{Rapport du SMB sur le dépistage par mammographie - Evaluation de Harry de Koning*}

L'analyse du rapport coût-efficacité effectuée dans le rapport du SMB sur le dépistage systématique par mammographie présente de graves lacunes à différents égards. Dans l'ensemble, le rapport sousestime les effets positifs du dépistage organisé, alors qu'il en surestime les effets négatifs. Les principaux manques sont les suivants:

- Pour évaluer le nombre de décès évités, le SMB ne s'appuie pas sur la statistique actuelle des décès par cancer du sein en Suisse, mais sur les données d'études randomisées datant des années 1980. Sur le plan méthodologique, il aurait été approprié d'utiliser les chiffres absolus actuels de façon analogue au groupe d'experts anglais dans leur rapport [3]. La démarche adoptée par le SMB conduit à sous-estimer les effets positifs en termes de décès évités.

- Les calculs du SMB couvrent une période de treize ans seulement. Sur le plan méthodologique, il aurait été approprié de tenir compte des effets du dépistage systématique sur toute la durée de vie des femmes. Les effets positifs du dépistage en termes d'années de vie gagnées compte tenu des décès évités sont ainsi fortement sous-estimés.

- Alors que les programmes de dépistage couvrent une période de vingt ans (ce qui correspond,

bonne qualité qui traduisent une approche différenciée. En plus des études randomisées qui, dans le cas présent, ne reflètent pas la réalité actuelle, il est nécessaire de tenir compte des observations faites par les programmes de dépistage - une exigence que le rapport du SMB sur le dépistage systématique par mammographie ne remplit pas.

D'autres rapports du SMB ont suscité des réactions similaires (cf. notamment le numéro 29/30 2014 du BMS [8]). Dans un article publié dans la Neue Zürcher Zeitung le 14 novembre 2013, Michael Schlander a pointé du doigt les insuffisances du SMB («Das Swiss Medical Board genügt nicht») [9]. Nous ne pouvons qu'appuyer les efforts de l'organe responsable en vue «d'améliorer tant les structures que les processus» [10], en espérant que ces réformes contribueront à la réalisation de HTA de bonne qualité en Suisse.

Vous souhaitez commenter cet article? II vous suffit pour cela d'utiliser la fonction «Ajouter un commentaire» dans la version en ligne. Vous pouvez également consulter les remarques de vos confrères sous: www.bullmed.ch/ numero-actuel/ articles-interactifs/

1 Swiss Medical Board. Dépistage systématique par mammographie. (2013). Publié le 2 février 2014.

2 Swiss Medical Board. Le traitement par statines en tant que prévention primaire des maladies cardiovasculaires. (2013). Publié le 2 avril 2014.

3 Independent UK Panel on Breast Cancer Screening (2012): The benefits and harms of breast cancer screening: an independent review. Lancet. 2012; 380: avec une mammographie tous les deux ans, à dix mammographies par femme), le SMB part, dans ses calculs, de trois mammographies par femme. Il sous-estime ainsi les effets positifs du dépistage systématique en termes d'années de vie gagnées.

- L'analyse coût-efficacité ne tient pas compte de tous les effets pertinents des programmes de dépistage; elle ne prend en particulier pas en considération le recul des diagnostics posés à un stade avancé.

- Le fait de se limiter à une période d'observation de treize ans amène à surestimer les répercussions négatives du dépistage liées aux surdiagnostics.

- Les répercussions négatives liées aux résultats faux-positifs sont surestimées car on part de I'hypothèse que la qualité de vie est fortement diminuée sur une période de plusieurs mois.

Harry de Koning, Professor of Evaluation of Screening, Erasmus MC, Rotterdam

Résumé de l'article 21st century science on the back of a coaster - A review of the Swiss Medical Board's 'Systematisches Mammographie-Screening' report (2014), by H.J. de Koning, MD PhD (submitted for publication)

4 Health Council of the Netherlands. Population screening for breast cancer: expectations and developments. The Hague: Health Council of the Netherlands; 2014. Publication no. 2014/01E. ISBN 978-94-6281-011-2

5 Swiss Medical Board. Organisation und Prozesse des Medical Board, état au 31 janvier 2011, consulté le 1er juillet 2014 sous www.medical-board.ch/fileadmin/ docs/public/mb/methodik/2011-01-31_Organisation_ und_Prozesse.pdf

6 Swiss Society of Senology. SMB Mammography Screening Recommendations: Concerns shared Conclusions wrong. Bull Méd Suisses. 2014;95:(36):1315-6.

7 Swiss Medical Board. Dépistage systématique par mammographie - Documentation et évaluation des prises de position concernant le rapport (Post-Publication-Review). Publié le 12 août 2014.

8 Arbeitsgruppe Lipide und Atherosklerose (AGLA), der Schweizerischen Gesellschaft für Kardiologie (SGK), und der Schweizerischen Gesellschaft für Herz- und Gefässchirurgie (SGHC). Statine zur Primärprävention kardiovaskulärer Erkrankungen. Bull Méd Suisses; 2014;95(29/30):1084-6 $1778-86$. medical-board-nicht-genuegt-1.18185108

10 Swiss Medical Board. Rapport annuel 2013.
9 www.nzz.ch/meinung/debatte/warum-das-swiss- 